\title{
Risk of delayed bleeding after hot snare polypectomy and endoscopic mucosal resection in the colorectum with continuation of anticoagulants
}

\author{
Takamasa Kobayashi $^{1}$, Manabu Takeuchi ${ }^{1}$, Yuki Hojo ${ }^{1}$, Yui Ishii ${ }^{1}$, Youhei Koseki ${ }^{1}$, Yoko Kobayashi ${ }^{1}$, \\ Motoi Azumi $^{1}$, Yuji Kobayashi ${ }^{1}$, Junji Kohisa ${ }^{1}$, Seiichi Yoshikawa ${ }^{1}$, Shuji Terai $^{2}$ \\ ${ }^{1}$ Division of Gastroenterology and Hepatology, Nagaoka Red Cross Hospital, Nagaoka, Japan; ${ }^{2}$ Division of Gastroenterology and Hepatology, \\ Niigata University Graduate School of Medical and Dental Sciences, Niigata, Japan \\ Contributions: (I) Conception and design: T Kobayashi, M Takeuchi, S Terai; (II) Administrative support: M Takeuchi, S Terai; (III) Provision of \\ study materials or patients: T Kobayashi, M Takeuchi, Y Hojo, Y Ishii, Y Koseki, Y Kobayashi, M Azumi, Y Kobayashi, J Kohisa, S Yoshikawa; (IV) \\ Collection and assembly of data: T Kobayashi, M Takeuchi; (V) Data analysis and interpretation: All authors; (VI) Manuscript writing: All authors; \\ (VII) Final approval of manuscript: All authors. \\ Correspondence to: Manabu Takeuchi, MD, PhD. Division of Gastroenterology and Hepatology, Nagaoka Red Cross Hospital, 2-297-1, Senshu, \\ Nagaoka, Niigata, 940-2085, Japan. Email: yasuzuka2000@yahoo.co.jp.
}

Background: Current guidelines recommend the temporary discontinuation of anticoagulants before colonoscopic polypectomy, but the effect of this practice on reducing the risk of delayed bleeding after hot snare polypectomy (HSP) and endoscopic mucosal resection (EMR) remains unclear. Our aim was to assess the impact of anticoagulants on the risk of colorectal delayed bleeding after HSP and EMR, and evaluate the necessity of drug withdrawal.

Methods: We reviewed the clinical data of patients with colorectal polyps using antithrombotic drugs who underwent HSP and/or EMR between January 2016 and September 2020 at Nagaoka Red Cross Hospital. After excluding antiplatelet users, patients were classified into those who continued anticoagulants [continuation group: 50 patients (93 lesions)] and those who discontinued anticoagulants [discontinuation group: 87 patients (190 lesions)].

Results: Delayed bleeding occurred in 12 lesions, and there was no significant difference in the incidence rates between the continuation and the discontinuation groups (3.2\% vs. 4.7\%; $\mathrm{P}=0.756)$. Logistic regression analysis showed that continued use of anticoagulants was not a significant risk factor for delayed bleeding compared to anticoagulant discontinuation (odds ratio, 0.670; 95\% CI, 0.177-2.537; P=0.556). There was no significant difference in the incidence rate and risk of delayed bleeding, regardless of the length of the anticoagulant withdrawal period.

Conclusions: Continued use of anticoagulants, compared to their discontinuation, did not increase the risk of colorectal delayed bleeding after HSP and EMR. Our results suggest that current guideline recommendations for anticoagulant withdrawal before colonoscopic polypectomy may be reconsidered.

Trial Registration: UMIN000040449.

Keywords: Anticoagulant; colorectum; endoscopic polypectomy; post-polypectomy bleeding

Submitted Jan 03, 2021. Accepted for publication Jun 08, 2021.

doi: 10.21037/jgo-21-3

View this article at: https://dx.doi.org/10.21037/jgo-21-3 


\section{Introduction}

Colorectal cancer is one of the most common malignancies and a major cause of cancer mortality worldwide (1). Early detection and treatment of colorectal cancer is important, and a screening colonoscopy with polypectomy has been revealed to be effective for decreasing colorectal cancer incidence and mortality (2,3). Endoscopic procedures, including hot snare polypectomy (HSP) and endoscopic mucosal resection (EMR), are commonly performed as minimally invasive treatments for resecting colorectal polyps. However, delayed post-polypectomy bleeding (DPPB) is one of the major complications of these procedures, with a reported incidence of $0.48-1.1 \%$ (4-6).

With the aging of society, the number of patients using anticoagulants for the prophylaxis or treatment of thromboembolic diseases is growing (7). Moreover, the number of cases of colonoscopic polypectomy in anticoagulant users is expected to increase. Thus, management of anticoagulants during the peri-endoscopic period aimed at optimizing the balance of risks between bleeding complications and thromboembolism is an important issue in clinical practice. Considering this situation, in 2014, the Japan Gastroenterological Endoscopy Society (JGES) published guidelines for gastroenterological endoscopy in patients undergoing antithrombotic treatment (8), with an appendix on anticoagulants, including direct oral anticoagulants (DOACs), in 2018 (9). In these guidelines, HSP and EMR are classified as procedures with a high risk of bleeding, and the withdrawal of anticoagulants or heparin bridging therapy (HBT) is recommended during the peri-endoscopic period. However, the efficacy of anticoagulant withdrawal in reducing the risk of colorectal DPPB has not been clarified. Moreover, previous studies have reported that HBT is associated with a higher risk of colorectal DPPB (10-13). Thus, little is known about the impact of anticoagulants on the risk of DPPB with respect to establishing an optimal strategy for colonoscopic polypectomy targeting anticoagulant users.

In this study, we aimed to assess the impact of anticoagulants on the risk of colorectal DPPB. We compared the risk of colorectal DPPB between continued and discontinued anticoagulant use during the periendoscopic period to evaluate the necessity of drug withdrawal as recommended in the JGES guidelines. We present the following article in accordance with the STROBE reporting checklist (Available at https://dx.doi. org/10.21037/jgo-21-3).

\section{Methods}

\section{Study subjects and data collection}

We reviewed the clinical data of patients who were using antithrombotic drugs and who underwent colonoscopic polypectomy for colorectal polyps between January 2016 and September 2020 at Nagaoka Red Cross Hospital in Nagaoka, Japan. Patients younger than 20 years old and those with lesions treated with endoscopic methods other than HSP or EMR [e.g., jumbo forceps polypectomy, hot forceps biopsy, and cold snare polypectomy (CSP)] were excluded from this study. The main outcome of this study was incidence of DPPB, and the following data were collected from the database: (I) patient characteristics included age, sex, comorbidities (hypertension, diabetes mellitus, dyslipidemia, need for hemodialysis, atrial fibrillation, deep vein thrombosis, cardiac valve replacement, congestive heart failure, prior stroke or transient ischemic attack, and ischemic heart disease), CHADS2 and CHA2DS2-VASc scores for patients with atrial fibrillation, number of lesions resected per colonoscopic procedure, status of treatment with antithrombotic drugs, and presence or absence of HBT and (II) lesion characteristics, including size, shape, location, histological type, achievement of complete resection, presence or absence of the prophylactic clipping procedure, and experience of treating endoscopist performing colonoscopies. DPPB was defined as hematochezia occurring within 30 days after the polypectomy and active bleeding or adherent clotting at the resection site, or blood pooling in the colorectal lumen as detected by emergency colonoscopy. The DPPB rate was calculated based on the total number of lesions treated. This study was conducted retrospectively and had a partially prospective design; patients and lesions between January 2016 and September 2019 were retrospectively reviewed and analyzed, and those between October 2019 and September 2020 were prospectively compiled. The study was approved by the ethics committee of Nagaoka Red Cross Hospital (No. 190929 and 200925) and listed in the UMIN clinical trials registry (UMIN000040449). All procedures performed in studies involving human participants were in accordance with the ethical standards of the institutional and/or national research committee and with the 1964 Helsinki Declaration and its later amendments or comparable ethical standards. Informed consent was obtained from all individual participants included in the study. 


\section{Endoscopic procedure}

As our institutional characteristics, most of procedures were performed by experienced endoscopists with performing more than 500 colonoscopies. Colonoscopies were performed using standard endoscopes (type CF-HQ290ZI, CF-HQ290I, PCF-H290ZI, or PCF-Q260JI; Olympus Co., Tokyo, Japan) after full bowel preparation. In cases of using magnifying scopes, magnifying observation and resection of lesions were performed in one procedure. The instruments used for resection were CAPTIVATOR ${ }^{\mathrm{TM}}$ (Boston Scientific Co., Natick, MA, USA), SnareMaster Plus (Olympus Co., Tokyo, Japan), and Dualoop (Medico's Hirata Inc., Osaka, Japan). Resection methods, including HSP and EMR, were appropriately selected for each lesion by the treating physician. The EMR technique consists of a local injection of $0.9 \%$ normal saline into the submucosal layer, followed by snaring and resection. In our institution, most of superficial or sessile-type lesions were treated with EMR, and HSP was mainly applied to resect pedunculated lesions. All lesions included in this study were treated with electrocautery procedures and electrosurgical units (ERBE ICC 200, Somo Technology Inc., Tokyo, Japan) set to $30 \mathrm{~W}$ for coagulation and cut mode. The decision to perform prophylactic clipping was made by the treating physician, with consideration for the prevention of DPPB, and hemostasis was confirmed at all resection sites at the end of the colonoscopy.

When DPPB was suspected because of hematochezia or other clinical symptoms and findings, emergency colonoscopy was performed to identify the bleeding site, and treatment with clipping, soft coagulation, or epinephrine-saline injection was administered.

\section{Management of anticoagulant drugs}

The anticoagulants were categorized into warfarin and DOACs, including dabigatran, rivaroxaban, apixaban, and edoxaban. The recommendations from the JGES guidelines for the management of anticoagulants in high-bleedingrisk procedures are as follows $(8,9)$ : (I) discontinuation of warfarin for 3-5 days with consideration of HBT before the procedure and (II) cessation of DOACs on the day of the procedure without HBT or discontinuation for 24-48 hours with consideration for HBT before the procedure. We consulted the physician that prescribed the anticoagulants regarding the safety of discontinuation. In accordance with the response (in cases of patients expected to have higher risk of thromboembolic diseases (e.g., higher CHADS2 and CHA2DS2-VASc scores) and those who treated with cardiac valve replacement, the physicians were not prone to recommend interruption of anticoagulants), the risk of hemorrhagic and thromboembolic events was explained, and the management of anticoagulants was determined in each patient after obtaining written informed consent. To evaluate the validity of the above methods recommended in the guidelines and the impact of anticoagulants on the risk of DPPB, we classified patients into the following groups: those who continued anticoagulants without anticoagulant withdrawal (continuation group) and those who discontinued anticoagulants before colonoscopy (for $\geq 1$ day, including the day of procedure) (discontinuation group) with or without HBT. Regarding HBT, continuous intravenous infusion of unfractionated heparin (UFH) was administered after the discontinuation of anticoagulants, and the dose of UFH was controlled to obtain an activated partial thromboplastin time (APTT) within 1.5-2.0-fold of the upper normal limit. UFH was stopped 6 hours before colonoscopy, and the normalization of APTT at the time of the procedure was checked. Regarding patients in the continuation group, to avoid the peak of DOAC blood concentration, we instructed those who scheduled to perform colonoscopy in AM to administrate anticoagulants after the procedure, and those who scheduled colonoscopy in $\mathrm{PM}$ to administrate in the morning. As for patients in the discontinuation group, if DPPB was not suspected upon the absence of hematochezia or other clinical symptoms and findings, anticoagulants were resumed on the next day after polypectomy. In patients taking warfarin, the prothrombin time-international normalized ratio (PTINR) before polypectomy was confirmed at $<3.0$, and UFH administration was restarted the next day until the PT-INR reached the therapeutic range.

\section{Statistical analysis}

Continuous variables are expressed as mean \pm standard deviation and were compared using Student's $t$-test, except for non-normally distributed variables, which are expressed as median (range) and were compared using the MannWhitney $U$-test. Categorical variables are expressed as number (percentage), and were compared using Fisher's exact test or the chi-squared test, as appropriate. A P value of $<0.05$ was considered statistically significant. The risk factors for DPPB were assessed using a logistic regression analysis and are expressed as odds ratios (ORs) and 95\% CIs. All statistical analyses were performed using SPSS, 


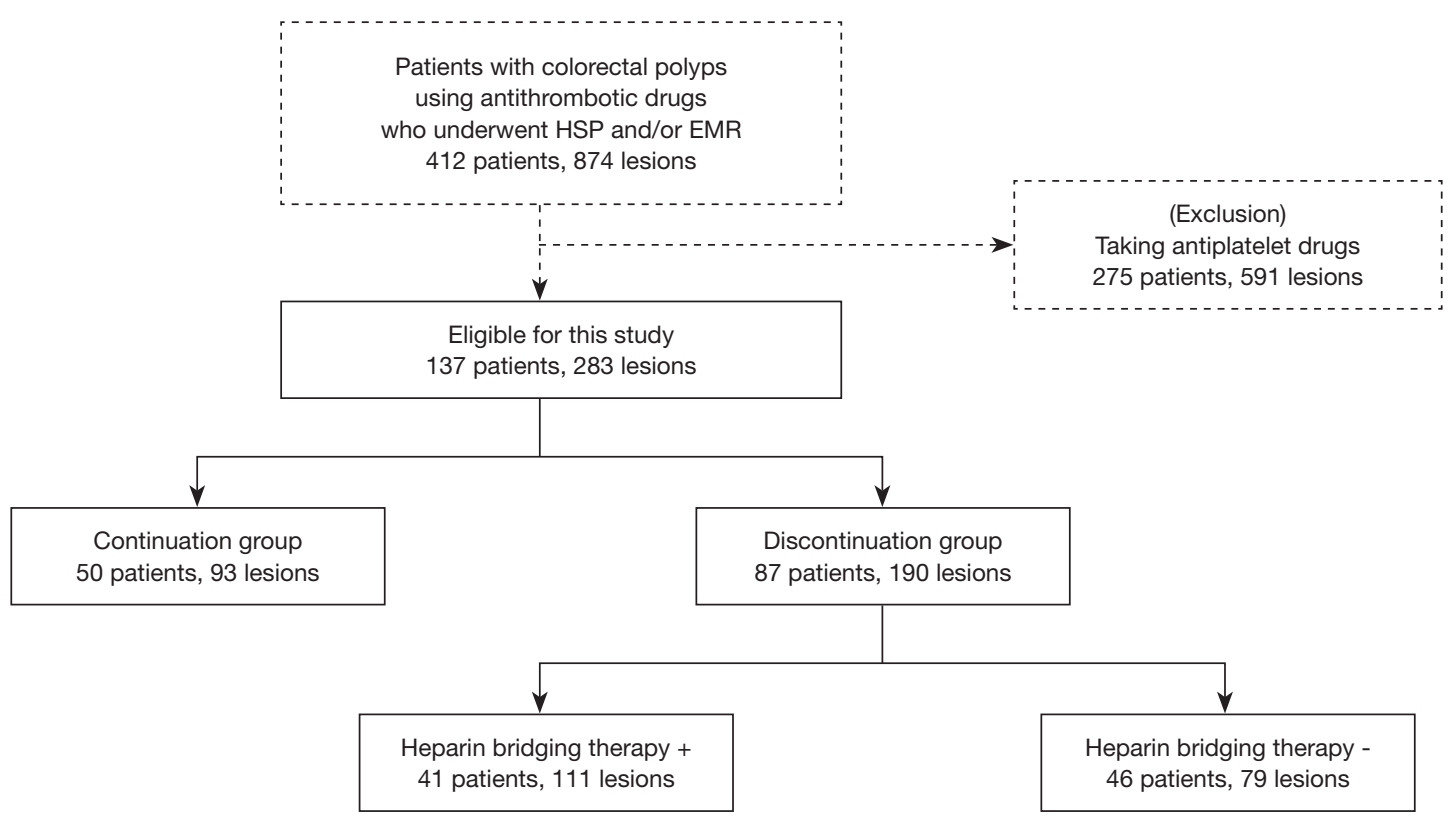

Figure 1 Flow diagram of the patients and lesions analyzed in this study. HSP, hot snare polypectomy; EMR, endoscopic mucosal resection.

version 26 (IBM Corp., Armonk, NY, USA).

\section{Results}

\section{Patients and lesions}

Figure 1 shows the flow diagram of the patients and lesions in this study. During the study period, 412 patients using antithrombotic drugs with 874 lesions were identified. Among the patients, 275 (591 lesions) who using antiplatelets were excluded to eliminate the impact of these drugs, leaving 137 patients with 283 lesions eligible for this study. The patients and lesions were classified into the continuation group [50 patients (93 lesions)] and the discontinuation group [87 patients (190 lesions)]. The discontinuation group comprised of 41 patients (111 lesions) with HBT and 46 patients (79 lesions) without HBT. Among them, 10 patients (12 lesions) experienced DPPB.

The clinical characteristics of the patients and lesions are shown in Table 1. Although many of the characteristics were similar between the groups, the proportion of DOACs prescribed was significantly different. Additionally, the discontinuation group had a significantly higher rate of lesions treated with prophylactic clipping than the continuation group. However, the DPPB rates for both groups were not significantly different $(3.2 \%$ vs. $4.7 \%$; $\mathrm{P}=0.756)$. No thromboembolic events occurred in any of the patients during the perioperative period.

Table 2 shows the univariate analysis for identifying the risk factors for DPPB in the continuation and discontinuation groups. The risk between continued and discontinued anticoagulant use was not significantly different (OR, 0.670; 95\% CI, 0.177-2.537; $\mathrm{P}=0.556$ ), and no other clinical variables were identified as significant risk factors for DPPB.

\section{Risk of DPPB in the discontinuation group with or without HBT}

Table 3 shows the comparison of the clinical characteristics of patients and lesions in the discontinuation group between the use and non-use of HBT. Of the 87 patients (190 lesions) in the discontinuation group, 41 (111 lesions) received HBT during the perioperative period. The proportion of men and the number of lesions treated per procedure were significantly higher in the discontinuation with HBT group. The proportion of DOAC users was significantly higher in the discontinuation without HBT group. The DPPB rate was not significantly different between the two groups $(4.5 \%$ vs. $5.1 \%$; $>>0.999)$.

Table 4 shows the univariate analysis for identifying the risk factors for DPPB in the discontinuation group. The use and non-use of HBT showed similar risks for DPPB (OR, 0.884; 95\% CI, 0.230-3.404; $\mathrm{P}=0.858$ ), and no other clinical 
Table 1 Characteristics of patients and lesions

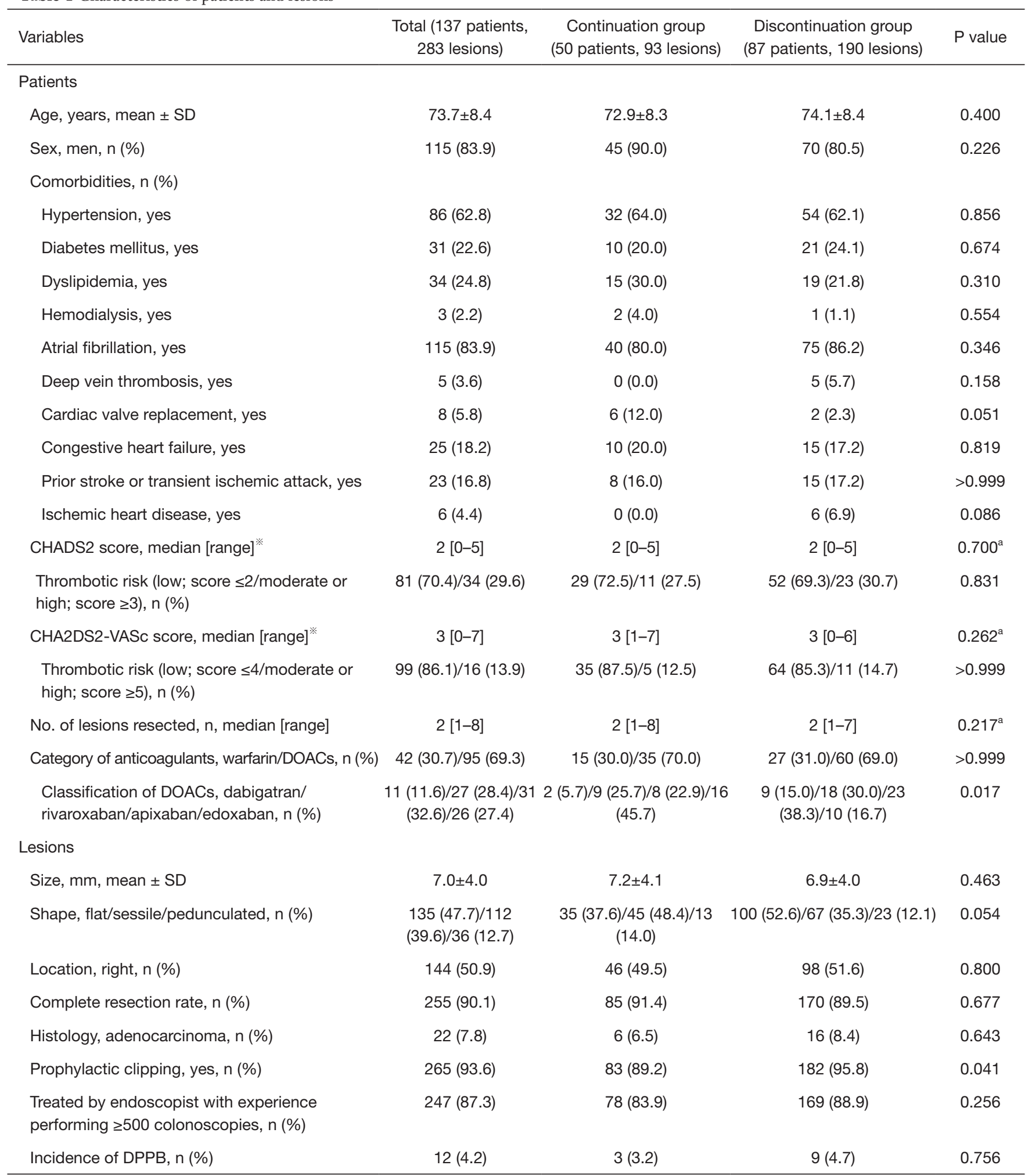

*, Patients with atrial fibrillation. ${ }^{\text {a }}$, Mann-Whitney $U$ test. SD, standard deviation; DOACs, direct oral anticoagulants; DPPB, delayed postpolypectomy bleeding. 
Table 2 Univariate analysis of the risk factor for delayed post-polypectomy bleeding

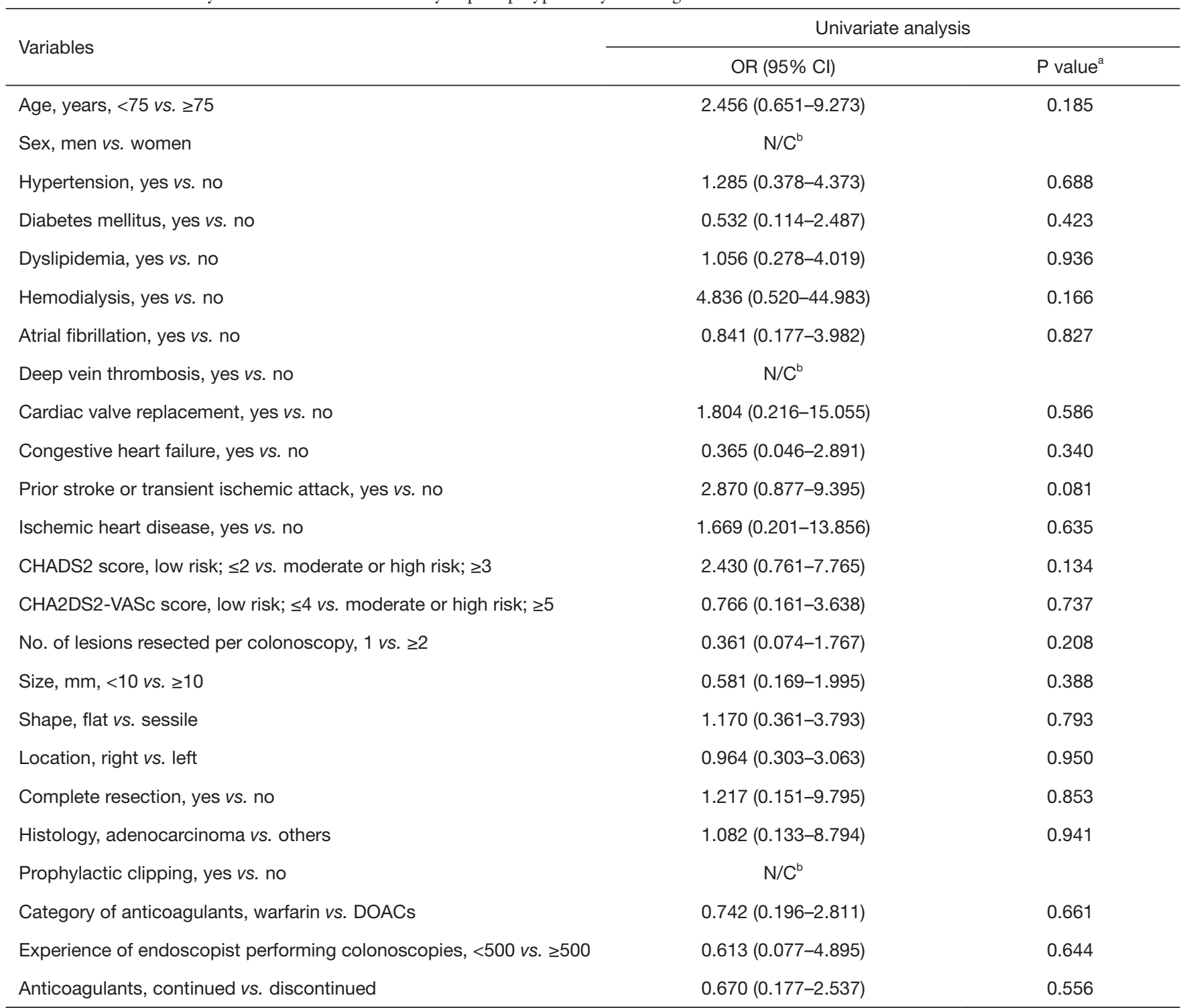

${ }^{\mathrm{a}}$, logistic regression analysis model was used. ${ }^{\mathrm{b}}$, the number of the lesions was too small and there were zero cell counts. OR, odds ratio; $\mathrm{Cl}$, confidence interval; N/C, not calculated; DOACs, direct oral anticoagulants.

variables were identified as significant risk factors for $\mathrm{DPPB}$.

\section{Clinical characteristics of patients and lesions with DPPB}

The clinical characteristics of the 10 patients (12 lesions) that experienced DPPB are shown in Table S1. Their median age was 73 years (range, 52 to 84 years). All of the patients were male, and all of the lesions were treated with prophylactic clipping. The period between polypectomy and DPPB ranged between 1 and 6 days. Emergency colonoscopies were performed for all the DPPB cases, with subsequent achievement of hemostasis, although 1 patient experienced rebleeding (case no. 2) and 3 patients required blood transfusions (case nos. 5, 9, and 10). The following anticoagulants were prescribed to these patients: warfarin (3 patients), dabigatran (2 patients), rivaroxaban (2 patients), apixaban ( 2 patients), and edoxaban (1 patient), and 3 patients received HBT. Among these patients, 1 case of DPPB occurred before anticoagulant therapy was restarted (case no. 1). One patient experienced DPPB after both 
Table 3 Comparison of characteristics of patients and lesions in the discontinuation group between with and without heparin bridging therapy

\begin{tabular}{|c|c|c|c|c|}
\hline Variables & $\begin{array}{l}\text { Total of discontinuation group } \\
\text { (87 patients, } 190 \text { lesions) }\end{array}$ & $\begin{array}{l}\text { Discontinuation with HBT } \\
\text { (41 patients, } 111 \text { lesions) }\end{array}$ & $\begin{array}{l}\text { Discontinuation without HBT } \\
\text { (46 patients, } 79 \text { lesions) }\end{array}$ & $P$ value \\
\hline \multicolumn{5}{|l|}{ Patients } \\
\hline Sex, men, n (\%) & $70(80.5)$ & $40(97.6)$ & $30(65.2)$ & $<0.001$ \\
\hline \multicolumn{5}{|l|}{ Comorbidities } \\
\hline Diabetes mellitus, yes, $\mathrm{n}(\%)$ & $21(24.1)$ & $13(31.7)$ & $8(17.4)$ & 0.139 \\
\hline Dyslipidemia, yes, n (\%) & $19(21.8)$ & $8(19.5)$ & $11(23.9)$ & 0.796 \\
\hline Hemodialysis, yes, n (\%) & $1(1.1)$ & $1(2.4)$ & $0(0.0)$ & 0.471 \\
\hline Congestive heart failure, yes, $\mathrm{n}(\%)$ & $15(17.2)$ & $6(14.6)$ & $9(19.6)$ & 0.583 \\
\hline $\begin{array}{l}\text { Prior stroke or transient ischemic attack, } \\
\text { yes, } n(\%)\end{array}$ & $15(17.2)$ & $9(22.0)$ & $6(13.0)$ & 0.395 \\
\hline Ischemic heart disease, yes, n (\%) & $6(6.9)$ & $3(7.3)$ & $3(6.5)$ & $>0.999$ \\
\hline CHADS2 score, median [range] ${ }^{*}$ & $2[0-5]$ & $2[0-5]$ & $2[0-5]$ & $0.991^{a}$ \\
\hline $\begin{array}{l}\text { Thrombotic risk (low; score } \leq 2 / \text { moderate } \\
\text { or high; score } \geq 3 \text { ), n (\%) }\end{array}$ & $52(69.3) / 23(30.7)$ & $24(66.7) / 12(33.3)$ & $28(71.8) / 11(28.2)$ & 0.803 \\
\hline CHA2DS2-VASc score, median [range] ${ }^{*}$ & $3[0-6]$ & $3[0-6]$ & $3[1-6]$ & $0.159^{\mathrm{a}}$ \\
\hline \multicolumn{5}{|l|}{ Lesions } \\
\hline Size, $m m$, mean $\pm S D$ & $6.9 \pm 4.0$ & $7.0 \pm 4.1$ & $6.7 \pm 3.8$ & 0.573 \\
\hline Shape, flat/sessile/pedunculated, n (\%) & $100(52.6) / 67(35.3) / 23(12.1)$ & $\begin{array}{c}60(54.1) / 34(30.6) \\
\quad / 17(15.3)\end{array}$ & $40(50.6) / 33(41.8) / 6(7.6)$ & 0.135 \\
\hline Location, right, n (\%) & $98(51.6)$ & $58(52.3)$ & $40(50.6)$ & 0.883 \\
\hline Complete resection rate, $\mathrm{n}(\%)$ & $170(89.5)$ & $98(88.3)$ & $72(91.1)$ & 0.635 \\
\hline Histology, adenocarcinoma, n (\%) & $16(8.4)$ & $8(7.2)$ & $8(10.1)$ & 0.598 \\
\hline Prophylactic clipping, yes, n (\%) & $182(95.8)$ & $106(95.5)$ & $76(96.2)$ & $>0.999$ \\
\hline $\begin{array}{l}\text { Treated by endoscopist with experience } \\
\text { performing } \geq 500 \text { colonoscopies, } n(\%)\end{array}$ & $169(88.9)$ & 99 (89.2) & 70 (88.6) & $>0.999$ \\
\hline Incidence of DPPB, n (\%) & $9(4.7)$ & $5(4.5)$ & $4(5.1)$ & $>0.999$ \\
\hline
\end{tabular}

*, Patients with atrial fibrillation. ${ }^{a}$, Mann-Whitney $U$ test. HBT, heparin bridging therapy; SD, standard deviation; DOACs, direct oral anticoagulants; DPPB, delayed post-polypectomy bleeding. 
Table 4 Univariate analysis of the risk factor for delayed post-polypectomy bleeding in the discontinuation group

\begin{tabular}{|c|c|c|}
\hline Variables & \multicolumn{2}{|c|}{ Univariate analysis } \\
\hline Age, years, $<75$ vs. $\geq 75$ & $3.031(0.613-14.988)$ & 0.174 \\
\hline Sex, men vs. women & $\mathrm{N} / \mathrm{C}^{\mathrm{b}}$ & \\
\hline Hypertension, yes vs. no & $1.415(0.343-5.837)$ & 0.631 \\
\hline Dyslipidemia, yes vs. no & $0.401(0.049-3.298)$ & 0.395 \\
\hline Hemodialysis, yes vs. no & $\mathrm{N} / \mathrm{C}^{\mathrm{b}}$ & \\
\hline Atrial fibrillation, yes vs. no & $1.342(0.161-11.179)$ & 0.786 \\
\hline Deep vein thrombosis, yes vs. no & $\mathrm{N} / \mathrm{C}^{\mathrm{b}}$ & \\
\hline Prior stroke or transient ischemic attack, yes vs. no & $2.418(0.622-9.395)$ & 0.202 \\
\hline Ischemic heart disease, yes vs. no & $1.491(0.174-12.790)$ & 0.716 \\
\hline CHADS2 score, low risk; $\leq 2$ vs. moderate or high risk; $\geq 3$ & $0.417(0.108-1.608)$ & 0.204 \\
\hline CHA2DS2-VASc score, low risk; $\leq 4$ vs. moderate or high risk; $\geq 5$ & $1.464(0.176-12.167)$ & 0.724 \\
\hline No. of lesions resected per colonoscopy, 1 vs. $\geq 2$ & $\mathrm{~N} / \mathrm{C}^{\mathrm{b}}$ & \\
\hline Size, $\mathrm{mm},<10$ vs. $\geq 10$ & $0.961(0.192-4.813)$ & 0.962 \\
\hline Shape, flat vs. sessile & $1.362(0.329-5.644)$ & 0.670 \\
\hline Location, right vs. left & $1.183(0.308-4.548)$ & 0.807 \\
\hline Heparin bridging therapy, yes vs. no & $0.884(0.230-3.404)$ & 0.858 \\
\hline
\end{tabular}

${ }^{\mathrm{a}}$, logistic regression analysis model was used. ${ }^{\mathrm{b}}$, the number of the lesions was too small and there were zero cell counts. OR, odds ratio; $\mathrm{Cl}$, confidence interval; N/C, not calculated; DOACs, direct oral anticoagulants.

UFH and warfarin were re-administered, and the results of coagulation tests when DPPB occurred were PT-INR of 1.39 and APTT of $84.3 \mathrm{~s}$ (case no. 4).

\section{Rate of DPPB in the continuation group by each kind of anticoagulant drug}

Figure 2 shows the rates of DPPB per lesion in the continuation group by each kind of anticoagulant drug. DPPB occurred only in patients taking warfarin and rivaroxaban, and the rate of DPPB was not significantly different between the patients with continued use of warfarin and DOACs $(\mathrm{P}=0.127)$. Of the DOACs used, there was no significant difference in the rates of DPPB by drug class $(\mathrm{P}=0.512)$.

\section{Risk of DPPB according to the anticoagulant withdrawal period}

To investigate the impact of the length of the anticoagulant 


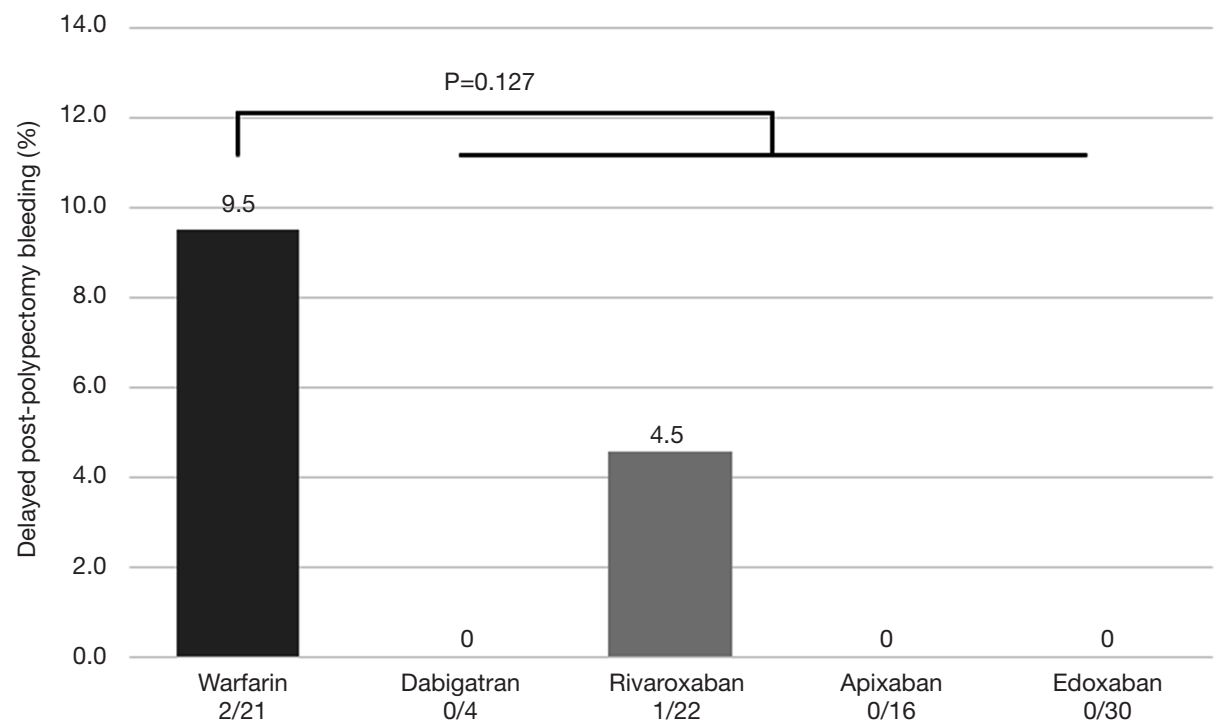

Figure 2 Rates of delayed post-polypectomy bleeding in the continuation group by each anticoagulant drug.

Table 5 The incidence rates of delayed post-polypectomy bleeding in the groups according to the withdrawal period of anticoagulants

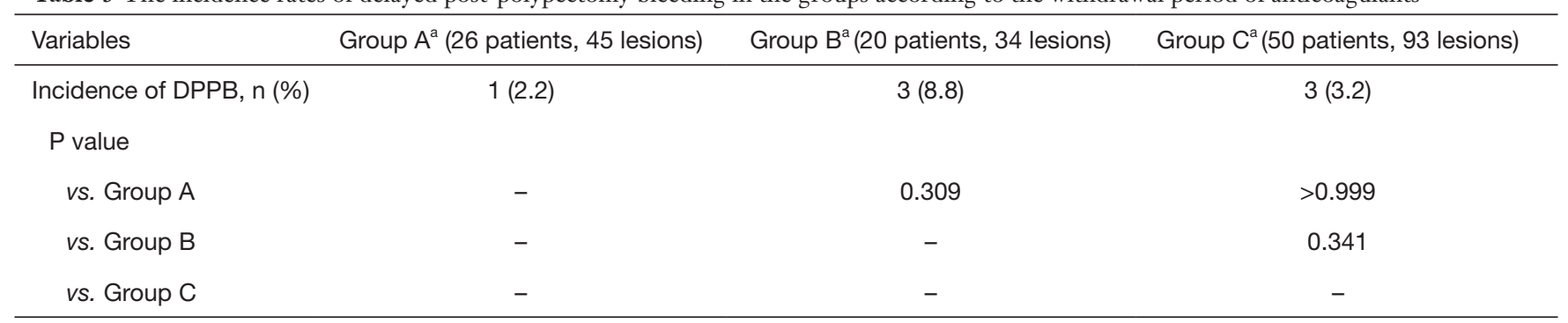

a, Group A, anticoagulant withdrawal period of $\geq 2$ days; Group B, anticoagulant withdrawal period of 1 day (on the day of colonoscopy);

Group C, without anticoagulant withdrawal period (continued). DPPB, delayed post-polypectomy bleeding.

withdrawal period on the risk of colorectal DPPB, anticoagulant users without HBT were classified into three groups as follows: patients with a withdrawal period of $\geq 2$ days (group A), those with a withdrawal period of 1 day (on the day of colonoscopy) (group B), and those without withdrawal (continued anticoagulants) (group C). Table 5 shows the incidence rates of DPPB in the above groups. There were 26 patients (45 lesions) in group A, 20 patients (34 lesions) in group B, and 50 patients (93 lesions) in group C. The incidence rates of DPPB in groups A, B, and C were $2.2 \%, 8.8 \%$, and $3.2 \%$, respectively, and there was no significant difference among these three groups. Table 6 shows the risk of DPPB according to the withdrawal period of anticoagulants, and the risk of colorectal DPPB was not significantly different in the univariate analysis, regardless of the length of the withdrawal period.

\section{Discussion}

In this study, we assessed the impact of anticoagulants on the risk of colorectal DPPB and evaluated the necessity of drug withdrawal during the peri-endoscopic period as recommended in the JGES guidelines. Our results showed that there was no significant difference in the incidence rates of DPPB between those who continued and discontinued anticoagulants, and the continued use of anticoagulants was not a significant risk factor for colorectal DPPB, compared to the discontinuation of anticoagulants.

To date, several efforts have been made to reduce the risk of colorectal DPPB for anticoagulant users. Replacement of warfarin with DOACs and temporary discontinuation before high-bleeding-risk endoscopic procedures are recommended in the JGES guidelines $(8,9)$. However, evidence regarding the effectiveness of anticoagulant 
Table 6 The risk assessment for delayed post-polypectomy bleeding in the groups according to the withdrawal period of anticoagulants

\begin{tabular}{lcc}
\hline & \multicolumn{2}{c}{ Univariate analysis } \\
\hline Group B vs. Group A & OR $(95 \%$ Cl) & P value \\
Group C vs. Group B & $4.258(0.423-42.870)$ & 0.219 \\
Group A vs. Group C & $0.344(0.066-1.796)$ & 0.206 \\
\hline
\end{tabular}

a, Group A, anticoagulant withdrawal period of $\geq 2$ days; Group B, anticoagulant withdrawal period of 1 day (on the day of colonoscopy); Group C, without anticoagulant withdrawal period (continued). ${ }^{\text {, }}$, logistic regression analysis model was used. DPPB, delayed postpolypectomy bleeding; OR, odds ratio; $\mathrm{Cl}$, confidence interval.

withdrawal for reducing the risk of colorectal DPPB has not been provided in the literature, while the increased risk of thromboembolism accompanying the interruption of anticoagulant treatment has been previously reported (14). In this study, we first evaluated the risk of colorectal DPPB according to the withdrawal period of anticoagulants. Although the incidence rate of colorectal DPPB in the group with 1-day withdrawal of anticoagulants (group B) was higher than the other groups, there was no significant difference among anticoagulant users classified according to the length of the withdrawal period. Univariate analysis also demonstrated that the withdrawal period of anticoagulants did not affect the risk of colorectal DPPB. Thus, the effect of the temporary discontinuation of anticoagulants for reducing the risk of colorectal DPPB was uncertain in this study.

To balance the risks between bleeding complications and thromboembolisms, HBT during the peri-endoscopic period is recommended in the JGES guidelines $(8,9)$. However, a previous randomized, placebo-controlled trial for warfarin users with atrial fibrillation demonstrated that HBT for elective surgery or other elective invasive procedures did not reduce the risk of arterial thromboembolism, whereas the incidence of major bleeding was increased (15). Regarding endoscopic polypectomy in the colorectum, some previous studies reported that HBT is associated with a higher risk of DPPB (10-13), and another recent study reported that the incidence rate of colorectal DPPB in the group of HSP with HBT was $12.0 \%$ (95\% CI, 5.0-19.1\%) (16). On the other hand, the results of our study showed no significant difference in the incidence rates and risks of colorectal DPPB between the use and non-use of HBT, and the incidence rate of DPPB in the discontinuation group with HBT was lower compared to those of previous studies. We estimate the reasons for these discrepancies as follows. First, the proportion of warfarin and DOACs; in some of the previous studies, the proportion of warfarin users in HBT group was relatively higher than that of ours [approximately 70-90\% (10-12) vs. 53.7\%, respectively]. In patients taking warfarin with HBT, both of warfarin and UFH were restarted on the next day of colonoscopic polypectomy and continued until the PT-INR reached the therapeutic range. Consequently, a higher proportion of warfarin users with HBT led to a longer period of $\mathrm{UFH}$ infusion and could relate to a higher risk of DPPB. Second, patients with concomitant use of antiplatelets were whether excluded or not; in our study, the patients receiving antiplatelet drugs were excluded to eliminate the impact of these drugs. However, previous studies included the patients with concomitant use of antiplatelets and analyzed altogether (10-12). Thus, it might additively affect the incidence of colorectal DPPB. Third, the incidence rate of DPPB was calculated based on the number of either patients or lesions; we calculate the incidence rate of DPPB based on the total number of lesions treated, whereas some previous studies have calculated it based on that of patients $(10-11,13)$. There is a controversy about the number of either patients or lesions should be used to calculate the incidence rate of DPPB. However, if calculated based on the number of patients, the incidence rate of DPPB would be higher owing to a lower denominator and the impact of HBT as risk factor would be emphasized. To evaluate the relationships between the bleeding risk and the characteristics of each lesion specifically, we consider that the assessment of the incidence rate of colorectal DPPB would be better analyzed per lesions (12). With respect to the impact of healthcare cost, the burden of colorectal polypectomy was substantially higher with HBT than with standard treatments (17). Although our results showed no significant difference in the incidence rates and risks of colorectal DPPB, the recommendation for HBT during the peri-endoscopic period seems to require reconsideration. 
The safety of uninterrupted antithrombotic drug therapy with respect to the risk of bleeding complications following colorectal polypectomy has been evaluated (18-21). However, most of them compared the bleeding risk of antithrombotic users with that of non-users. Generally, clinical characteristics, such as age, sex, and comorbidities, tend to differ between patients with and without antithrombotic treatments. Additionally, the resumption of antithrombotic drugs after colonoscopic polypectomy can influence the risk of DPPB (22). Thus, the risk of continued antithrombotic medication usage would be better evaluated by comparing it with temporary discontinuation. A recent randomized, placebo-controlled trial for clopidogrel users showed that the risk of colorectal DPPB was not significantly different between drug interruption and non-interruption (23). For anticoagulants, a retrospective observational study of warfarin users reported that the incidence rate of colorectal DPPB without interruption of anticoagulation was $0 \%(0 / 41$ lesions) (24). Although the incidence rate of our continuation group was higher especially in warfarin users [9.5\% (2/21)], the value was rather close to that of another recent study $[12.2 \%(5 / 41)](25)$ and there was no significant difference compared to that of DOAC users. On the other hand, another retrospective study reported that the incidence rate of colorectal post-polypectomy bleeding was significantly higher in those with continued anticoagulant use (including warfarin and DOAC users) than in those with anticoagulant discontinuation and HBT non-use (26). However, patients with concomitant antiplatelet treatments (including lowdose aspirin, thienopyridine, etc.) were not excluded in the study protocol. Thus, these other drugs might have influenced their findings. To our knowledge, ours is the first study that included both warfarin and DOAC users and excluded antiplatelet users to compare the risk of colorectal DPPB between the continuation and discontinuation of anticoagulants.

We consider that there are some merits of colonoscopic polypectomy with continuation of anticoagulants as follows. First, patients recommended not to interrupt anticoagulants by prescribing physicians become targets of performing colonoscopic polypectomy. Second, in cases of patients with difficulty in getting agreement of anticoagulant withdrawal (for reasons such as afraid of thromboembolic diseases, etc.), they can undergo treatment for colorectal lesions. Third, if patients fail to stop anticoagulants on the day of colonoscopy for some reasons and colorectal lesions eligible for colonoscopic polypectomy are detected, we can avoid to plan secondary colonoscopy on another day. Besides, if anticoagulant therapy were stopped and thromboembolic event unfortunately occurred during the peri-endoscopic period, it would be difficult to rule out the causal relationship between them. Therefore, we consider that if the bleeding rate is similar, colonoscopic polypectomy with continuation of anticoagulants is a better strategy than the management recommended in the present guidelines.

This study had several limitations. First, this was a single-institutional study, and the total number of patients and lesions was relatively small. Although approximately 400 lesions in each group are required to prove equivalence and non-inferiority between the continuation and the discontinuation groups (at least $80 \%$ power to show non-inferiority at a margin of $10 \%$ ), it was difficult to collect such number of sample size. Second, our study was observational and mostly retrospective in design, and the characteristics of patients and lesions were not matched between each group. In previous studies, some clinical factors related to patients and lesions have been demonstrated as risk factors for colorectal DPPB. However, in our study, none of them were detected as significant risk factors and some of them could not be calculated in the univariate analysis. Third, the lesion size of our study was relatively small to treat with HSP and EMR. CSP is recommended to treat colorectal lesions less than $9 \mathrm{~mm}$ with high confidence of benign nature and reported to have lower risk of bleeding complications $(27,28)$. However, in our institution, resection of colorectal lesion with CSP was initiated to perform in 2016, and a substantial proportion of subcentimeter lesions were treated with HSP and EMR especially in the beginning of study period. The bleeding risk of HSP and EMR would better to be evaluated for colorectal lesion larger than $10 \mathrm{~mm}$ or with a high suspicion of malignancy. Fourth, we could not evaluate the impact on the risk of colorectal DPPB by each kind of anticoagulant drug class in detail owing to our small sample size. Although our results showed no significant differences in the rate and risk of colorectal DPPB between warfarin and DOACs, there are differences in pharmacological mechanisms between them (as well as among DOACs including dabigatran, rivaroxaban, apixaban, and edoxaban). Hence, it would be desirable to evaluate the impact on colorectal DPPB by patients using each kind of anticoagulant drug. Finally, no patient experienced thromboembolic events during the peri-endoscopic period; thus, we could not assess the risk of thromboembolic events according to anticoagulant use. We suggest that a multicenter surveillance, including a larger number of case-matched 
patients and lesions appropriate for HSP and EMR is necessary to assess the impact of anticoagulants on the risk of colorectal DPPB.

In conclusion, the incidence rate and risk of DPPB was similar between those who continued and discontinued anticoagulants. Additionally, the length of the anticoagulant withdrawal period did not affect the risk of colorectal DPPB. Our results suggest that the continued use of anticoagulants might be acceptable for colorectal polypectomy, and that the managements recommended in the present guidelines may be reconsidered.

\section{Acknowledgments}

Funding: None.

\section{Footnote}

Reporting Checklist: The authors have completed the STROBE reporting checklist. Available at https://dx.doi. org/10.21037/jgo-21-3

Data Sharing Statement: Available at https://dx.doi. org/10.21037/jgo-21-3

Peer Review File: Available at https://dx.doi.org/10.21037/ jgo-21-3

Conflicts of Interest: All authors have completed the ICMJE uniform disclosure form (Available at https://dx.doi. org/10.21037/jgo-21-3). The authors have no conflicts of interest to declare.

Ethical Statement: The authors are accountable for all aspects of the work in ensuring that questions related to the accuracy or integrity of any part of the work are appropriately investigated and resolved. This study was approved by the ethics committee of Nagaoka Red Cross Hospital (approval number 190929 and 200925). All procedures performed in this study involving human participants were in accordance with the ethical standards of the institutional and/or national research committee and with the 1964 Helsinki Declaration and its later amendments or comparable ethical standards. Informed consent was obtained from all individual participants included in the study.

Open Access Statement: This is an Open Access article distributed in accordance with the Creative Commons Attribution-NonCommercial-NoDerivs 4.0 International License (CC BY-NC-ND 4.0), which permits the noncommercial replication and distribution of the article with the strict proviso that no changes or edits are made and the original work is properly cited (including links to both the formal publication through the relevant DOI and the license). See: https://creativecommons.org/licenses/by-nc-nd/4.0/.

\section{References}

1. Bray F, Ferlay J, Soerjomataram I, et al. Global cancer statistics 2018: GLOBOCAN estimates of incidence and mortality worldwide for 36 cancers in 185 countries. CA Cancer J Clin 2018;68:394-424.

2. Winawer SJ, Zauber AG, Ho MN, et al. Prevention of colorectal cancer by colonoscopic polypectomy. The National Polyp Study Workgroup. N Engl J Med 1993;329:1977-81.

3. Nishihara R, Wu K, Lochhead P, et al. Long-term colorectal-cancer incidence and mortality after lower endoscopy. N Engl J Med 2013;369:1095-105.

4. Levin TR, Zhao W, Conell C, et al. Complications of colonoscopy in an integrated health care delivery system. Ann Intern Med 2006;145:880-6.

5. Watabe H, Yamaji Y, Okamoto M, et al. Risk assessment for delayed hemorrhagic complication of colonic polypectomy: polyp-related factors and patient-related factors. Gastrointest Endosc 2006;64:73-8.

6. Choung BS, Kim SH, Ahn DS, et al. Incidence and risk factors of delayed postpolypectomy bleeding: a retrospective cohort study. J Clin Gastroenterol 2014;48:784-9.

7. Benjamin EJ, Virani SS, Callaway CW, et al. Heart Disease and Stroke Statistics-2018 Update: A Report From the American Heart Association. Circulation 2018;137:e67-e492.

8. Fujimoto K, Fujishiro M, Kato M, et al. Guidelines for gastroenterological endoscopy in patients undergoing antithrombotic treatment. Dig Endosc 2014;26:1-14.

9. Kato M, Uedo N, Hokimoto S, et al. Guidelines for Gastroenterological Endoscopy in Patients Undergoing Antithrombotic Treatment: 2017 Appendix on Anticoagulants Including Direct Oral Anticoagulants. Dig Endosc 2018;30:433-40.

10. Inoue T, Nishida T, Maekawa A, et al. Clinical features of post-polypectomy bleeding associated with heparin bridge therapy. Dig Endosc 2014;26:243-9. 
11. Kubo T, Yamashita K, Onodera K, et al. Heparin bridge therapy and post-polypectomy bleeding. World J Gastroenterol 2016;22:10009-14.

12. Ishigami H, Arai M, Matsumura T, et al. Heparinbridging therapy is associated with a high risk of postpolypectomy bleeding regardless of polyp size. Dig Endosc 2017;29:65-72.

13. Fujita M, Murao T, Osawa M, et al. Colonic endoscopic mucosal resection in patients taking anticoagulants: Is heparin bridging therapy necessary? J Dig Dis 2018;19:288-94.

14. Blacker DJ, Wijdicks EF, McClelland RL. Stroke risk in anticoagulated patients with atrial fibrillation undergoing endoscopy. Neurology 2003;61:964-8.

15. Douketis JD, Spyropoulos AC, Kaatz S, et al. Perioperative Bridging Anticoagulation in Patients with Atrial Fibrillation. N Engl J Med 2015;373:823-33.

16. Takeuchi Y, Mabe K, Shimodate Y, et al. Continuous Anticoagulation and Cold Snare Polypectomy Versus Heparin Bridging and Hot Snare Polypectomy in Patients on Anticoagulants With Subcentimeter Polyps: A Randomized Controlled Trial. Ann Intern Med 2019;171:229-37.

17. Hayashi S, Nakamatsu D, Matsubara T, et al. Burden of health-care costs for patients with heparin replacement for colorectal EMR in Japan. Pharmacol Res Perspect 2017;5:e00366.

18. Singh M, Mehta N, Murthy UK, et al. Postpolypectomy bleeding in patients undergoing colonoscopy on uninterrupted clopidogrel therapy. Gastrointest Endosc 2010;71:998-1005.

19. Feagins LA, Iqbal R, Harford WV, et al. Low rate of postpolypectomy bleeding among patients who continue thienopyridine therapy during colonoscopy. Clin

Cite this article as: Kobayashi T, Takeuchi M, Hojo Y, Ishii Y, Koseki Y, Kobayashi Y, Azumi M, Kobayashi Y, Kohisa J, Yoshikawa S, Terai S. Risk of delayed bleeding after hot snare polypectomy and endoscopic mucosal resection in the colorectum with continuation of anticoagulants. J Gastrointest Oncol 2021;12(4):1518-1530. doi: 10.21037/jgo-21-3
Gastroenterol Hepatol 2013;11:1325-32.

20. Shibuya T, Nomura O, Kodani T, et al. Continuation of antithrombotic therapy may be associated with a high incidence of colonic post-polypectomy bleeding. Dig Endosc 2017;29:314-21.

21. Yu JX, Oliver M, Lin J, et al. Patients Prescribed Direct-Acting Oral Anticoagulants Have Low Risk of Postpolypectomy Complications. Clin Gastroenterol Hepatol 2019;17:2000-2007.e3.

22. Sawhney MS, Salfiti N, Nelson DB, et al. Risk factors for severe delayed postpolypectomy bleeding. Endoscopy 2008;40:115-9.

23. Chan FKL, Kyaw MH, Hsiang JC, et al. Risk of Postpolypectomy Bleeding With Uninterrupted Clopidogrel Therapy in an Industry-Independent, DoubleBlind, Randomized Trial. Gastroenterology 2019;156:91825.e1.

24. Friedland S, Soetikno R. Colonoscopy with polypectomy in anticoagulated patients. Gastrointest Endosc 2006;64:98-100.

25. Shimodate Y, Ueno M, Sunami T, et al. Post-polypectomy bleeding in hot-snare polypectomy of colonic polyps under continued warfarin or short interruption of direct oral anticoagulants. Int J Colorectal Dis 2019;34:1705-12.

26. Yanagisawa N, Nagata N, Watanabe K, et al. Postpolypectomy bleeding and thromboembolism risks associated with warfarin vs direct oral anticoagulants. World J Gastroenterol 2018;24:1540-9.

27. Deenadayalu VP, Rex DK. Colon polyp retrieval after cold snaring. Gastrointest Endosc 2005;62:253-6.

28. Repici A, Hassan C, Vitetta E, et al. Safety of cold polypectomy for $<10 \mathrm{~mm}$ polyps at colonoscopy: a prospective multicenter study. Endoscopy 2012;44:27-31. 
Table S1 Clinical characteristics of 10 patients with 12 lesions experienced delayed post-polypectomy bleeding

\begin{tabular}{|c|c|c|c|c|c|c|c|c|c|c|c|c|c|c|}
\hline \multirow{2}{*}{ Case No. } & \multirow{2}{*}{$\begin{array}{l}\text { Age, } \\
\text { years }\end{array}$} & \multirow{2}{*}{ Sex } & \multirow{2}{*}{ Comorbidities } & \multicolumn{4}{|c|}{ Lesion characteristics } & \multirow{2}{*}{$\begin{array}{l}\text { Prophylactic } \\
\text { Clipping }\end{array}$} & \multirow{2}{*}{ Anticoagulants } & \multirow{2}{*}{$\begin{array}{l}\text { Drug withdrawal } \\
\text { period, day }\end{array}$} & \multirow{2}{*}{$\begin{array}{l}\text { Heparin bridging } \\
\text { therapy }\end{array}$} & \multirow{2}{*}{$\begin{array}{l}\text { Bleeding date after } \\
\text { polypectomy, day }\end{array}$} & \multirow{2}{*}{ Rebleeding } & \multirow{2}{*}{$\begin{array}{c}\text { Blood } \\
\text { transfusion }\end{array}$} \\
\hline & & & & Size, $\mathrm{mm}$ & Shape & Location & Histology & & & & & & & \\
\hline 1 & 84 & M & $\mathrm{HT}, \mathrm{Af}, \mathrm{CHF}$ & 12 & Sessile & Left & Adenocarcinoma & Yes & Dabigatran & 1 & no & 1 & No & No \\
\hline \multirow[t]{3}{*}{2} & 74 & M & $\mathrm{HT}$, Af, prior stroke & 5 & Flat & Right & Adenoma & Yes & Apixaban & 2 & yes & 2 & Yes & No \\
\hline & & & & 3 & Flat & Right & Adenoma & Yes & & & & & & \\
\hline & & & & 5 & Flat & Left & Adenoma & Yes & & & & & & \\
\hline 3 & 74 & M & ischemic heart disease & 3 & Flat & Right & Adenoma & Yes & Rivaroxaban & 2 & yes & 2 & No & No \\
\hline 4 & 61 & M & HT, DL, Af & 6 & Sessile & Left & Adenoma & Yes & Warfarin & 3 & yes & 6 & No & No \\
\hline 6 & 76 & M & $\mathrm{HT}, \mathrm{DM}, \mathrm{Af}$, prior stroke & 4 & Flat & Left & Adenoma & Yes & Dabigatran & 1 & no & 3 & No & No \\
\hline 7 & 67 & M & Af & 10 & Flat & Right & Adenoma & Yes & Edoxaban & 1 & no & 2 & No & No \\
\hline 8 & 67 & M & Af & 5 & Flat & Left & Adenoma & Yes & Rivaroxaban & 0 (continued) & no & 1 & No & No \\
\hline 9 & 78 & M & HT, DM, DL, HD, Af, prior stroke & 12 & Sessile & Right & Adenoma & Yes & Warfarin & 0 (continued) & no & 3 & No & Yes \\
\hline 10 & 52 & M & cardiac valve replacement & 15 & Sessile & Left & Adenoma & Yes & Warfarin & 0 (continued) & no & 2 & No & Yes \\
\hline
\end{tabular}

$\mathrm{HT}$, hypertension; Af, atrial fibrillation; CHF, congestive heart failure; DL, dyslipidemia; DM, diabetes mellitus; HD, hemodialysis. 